\title{
CAPTURING VOLUNTARY, INVOLUNTARY, AND HABITUAL COMPONENTS OF DRIVER DISTRACTION IN A SELF-REPORTED QUESTIONNAIRE
}

\author{
Susana Marulanda, Huei-Yen Winnie Chen, Birsen Donmez \\ University of Toronto \\ Toronto, ON, CA \\ donmez@mie.utoronto.ca
}

\begin{abstract}
Summary: To maximize the effectiveness of strategies for mitigating driver distraction, it is crucial to understand the factors underlying drivers' engagement in distractions. This article describes a step toward an improved version of the Susceptibility to Driver Distraction Questionnaire (SDDQ), namely the development of an exploratory questionnaire based on findings from the original SDDQ. In this exploratory questionnaire, the Theory of Planned Behaviour continues to serve as the framework for investigating voluntary distractions, relating intentional actions to attitudes, perceived behavioural control, and perceived social norms regarding distractions. Involuntary distractions are captured by investigating the difficulty associated with ignoring information that is not critical for safe driving. A new component of habitual behaviours is also added to measure distractions that involve minimal conscious control, yet were once intentional and goal-driven. The resulting exploratory questionnaire will be used in an upcoming online survey study to determine the items that most effectively capture voluntary, involuntary, and habitual distraction. An improved SDDQ will be generated based on analyses of this pending study.
\end{abstract}

\section{INTRODUCTION}

Driver distraction has been identified as a major contributor to vehicle crashes (Klauer et al., 2014). On-going research efforts are still trying to understand causes of driver distraction and ways to mitigate associated risks. Our own efforts in understanding driver distraction at the individual driver level has led us to develop the Susceptibility to Driver Distraction Questionnaire (SDDQ), a 39-item self-reported data collection tool that distinguishes between voluntary distraction resulting from a driver's intentional engagement in a secondary task (e.g., sending a text message), and involuntary distraction resulting from a driver's inability to suppress stimuli irrelevant to driving (e.g., the ringing of a cell phone that the driver does not intend to answer) (Feng, Marulanda, \& Donmez, 2014). Self-reported measures have been used to examine driver distraction and some of the underlying motivations for engaging in distractions while driving (McEvoy, Stevenson, \& Woodward, 2006; Schroeder, Meyers, \& Kostyniuk, 2013). However, to our knowledge, no self-reported measure has been developed to distinguish between voluntary and involutary distraction, which is crucial for understanding how individuals may differ in their susceptibility to driver distraction.

SDDQ collects data on: (1) frequency of engagement in distractions while driving; (2) attitudes and beliefs toward voluntary distraction; and (3) susceptibility to involuntary distraction, which probes drivers' inability to suppress distracting stimuli while driving. Items in (1) and (2) are surveyed with respect to six distractions: hold phone conversations, manually interact with a 
phone (e.g., sending text messages), adjust the settings of in-vehicle technology (e.g., radio channel or song selection), read roadside advertisements, continually check roadside accident scenes if there are any, and chat with passengers if there are any. An additional item of 'daydream' is also included in (1). The involuntary distraction section probes respondents on how distracting they find eight different items while driving: a ringing phone, an audio cell phone alert, music, talk shows on the radio, roadside advertisements, roadside accident scenes, passengers' conversations, and daydreaming.

SDDQ has fared well against known scales of risky driver behaviours, personality, and cognitive limitations (Feng et al., 2014). The subscales on social-psychological factors (i.e., attitude, social norms, and behavioural control) have also been found useful in predicting self-reported engagement in voluntary distractions (Chen, Donmez, Hoekstra-Atwood, \& Marulanda, in review). Furthermore, the internal consistency of SDDQ is demonstrated as satisfactory, although the test-retest reliability of some of its subscales ranges from poor to fair (Marulanda, Chen, \& Donmez, in press). While SDDQ has demonstrated potential for studying driver distraction, we acknowledge some limitations in the existing questionnaire: the selection of distractions probed and the number of questions in each subscale are limited, making it difficult to assess construct validity. In addition, there are concerns over the measure of perceived social norms (there may not be social norms associated with distractions such as 'read roadside advertisements') and involuntary distraction (how well one recalls involuntary distraction experience over time is unclear). Furthermore, the driving context is not provided in SDDQ and some items may not be specific enough to elicit useful and consistent responses. Finally, SDDQ does not include measures of habitual behaviour, which belongs to neither voluntary nor involuntary engagement, but has also been identified as an important factor in driver distraction (Bayer \& Campbell, 2012).

SDDQ has gone through major revisions in consideration of the issues identified. This paper presents an overview of these revisions, focusing on the theoretical underpinnings of various subscales. The resulting 400-item questionnaire is exploratory in nature. Data collected from this questionnaire will undergo further analysis to determine the best set of items for measuring susceptibility to voluntary, inolvuntary, and habitual distractions.

\section{VOLUNTARY ENGAGEMENT IN DRIVER DISTRACTION}

In $2010,41 \%$ of drivers in the United States reported using cell phones to make or receive calls during at least some of their driving trips, but only 17\% reported using some type of hands-free device when doing so (Tison, Chaudhary, \& Cosgrove, 2011). Although the percentage of drivers who report sending text messages while driving is much smaller than the percentage of those who report holding phone conversations, the trend shows a slight increase from $12 \%$ in 2010 to $14 \%$ in 2012 (Schroeder et al., 2013). Aside from cell phone use, 8\% of drivers reported using a navigation system for directions, 11\% reported eating or drinking while driving, and 49\% reported always or almost always talking to a passenger while driving (Schroeder et al., 2013). These activities are also part of the coding scheme of driver distraction for National Highway Traffic Safety Administration's (NHTSA) crash databases: Fatality Analysis Reporting System (FARS) and General Estimates System (GES) (National Highway Traffic Safety Administration, 2014). 
The exploratory survey intends to measure a representative sample of voluntary driver distraction that not only consists of the most prevalent distractions currently known, but also those that represent different modalities where interference with driving may occur. As driving is primarily a visual-motor task, it is most susceptible to interference from tasks that require drivers to take their eyes off the road and/or hands off the steering wheel. In fact, data from the 100-car naturalistic study showed that almost 80 percent of all crashes and 65 percent of all near-crashes occurred when drivers looked away from the forward roadway (Dingus et al., 2006). Driving performance can also be degraded by cognitive interference, which occurs when attention is directed elsewhere from the mental processes required for safe driving (Strayer, Watson, \& Drews, 2011) and may explain the disruptive effects of hands-free cell phone conversations on driving performance (Strayer, Drews, \& Johnston, 2003). The final selection of distractions to use in the current survey aims to capture tasks that impose varying degrees of demands on drivers' input and output modalities, working memory, and cognitive resources:

- Dial a number on a cell phone manually / with voice command

- Read / send a text message on a cell phone

- Read email on a cell phone or tablet

- Enter destination on a mounted / non-mounted navigational device

- Adjust in-vehicle systems manually / with voice commands

- Update or check social media (e.g., Facebook, Instagram, or Twitter) on a cell phone or tablet

- Drink a hot beverage

- Personal grooming

- Talk to passengers if any

- Listen to any audio entertainment

- Phone conversation on a hand-held device / on a hands-free device

\section{Theory of Planned Behaviour}

The current questionnaire continues to rely on the Theory of Planned Behaviour (TPB; Ajzen, 1991) for investigating voluntary engagement in driver distraction. TPB stipulates that performance of a behaviour is determined by an individual's intention, which in turn is determined by the individual's attitudes, perceived behavioural control, and perceived social norms surrounding the behaviour (Ajzen, 1991). In the original SDDQ, each of these TPB constructs were assessed using only one generic statement, repeated for six distraction items surveyed. The current version uses multiple questions and rating scales to measure each construct, providing a more comprehensive assessment of the construct.

Attitude reflects an individual's favourable or unfavourable evaluation of performing a behaviour (Ajzen, 1991). The current questionnaire replaces the original Likert scale rating of a generic statement about attitudes ("I think it is alright to drive and engage in...") with semantic differential scales over specific qualities that may be associated with distracted driving. For each distraction item probed, the respondent is asked to choose their position on a scale of 1 to 5 between the paired bipolar adjectives: 'safe' versus 'dangerous', 'pleasant' versus 'unpleasant', and 'wise' versus 'unwise'. Further, the value of convenience, a potential benefit that drivers may associate with engaging in the secondary task (e.g., "it is good use of my time to drive and groom"), and an explicit evaluation of the alternative behaviour (e.g., "I lose respect for people 
who drive and send text messages on their mobile phone") are probed using 5-point Likert scales, ranging from strongly disagree to strongly agree with each statement.

Perceived Behavioural Control. The new questionnaire differentiates two kinds of perceived behavioural control: self-efficacy and controllability. Self-efficacy is measured by the perceived difficulty of engaging in distractions while driving. An example item in the original SDDQ associated with this construct was: "You believe you can drive well even when you hold phone conversations." Controllability refers to self-assessment of the driver's ability to control if and when they engage in distractions while driving, using items such as "I decide whether I drive and dial a phone number using voice commands."

Descriptive and Injunctive Norms. We continue to distinguish between descriptive and injunctive norms. Descriptive norms refer to how individuals perceive other drivers behave on the road (e.g., "most drivers drive and at the same time read their email on a mobile phone or tablet"). Injunctive norms refer to individuals' perceived approval by those who are important to them (e.g., "people who are important to me would approve of me driving and at the same time holding phone conversation on a hand-held device"). In addition, to address the issue of low testretest reliability among items in the injunctive subscale of SDDQ (Marulanda et al., in press), distractions that were unlikely to have strong social norms attached to them were not incorporated in the exploratory questionnaire. These distractions include: read roadside advertisements, visually dwell on roadside accident scenes, adjust the audio system using controls on the console/voice commands, chat with passengers, listen to audio entertainment, drink a hot beverage, and personal grooming.

\section{Scenarios}

Drivers' decision to engage in a non-driving task and the amount of attentional capacity they may have in dealing with stimuli in the driving environment largely depend on the context, which may influence the level of perceived risk associated with engaging in a secondary task, and the amount of stimuli present in the environment. For example, drivers report being less willing to answer a phone call when approaching a turning manoeuvre (e.g., U-turn or left turn) than when stopped at a traffic signal (Lerner \& Boyd, 2005). Providing context is therefore extremely useful in supporting drivers to answer questions about their distraction engagement and attentional capacities more accurately. In the new questionnaire, we do so by generating two different scenarios that will examine driver responses across high and low workload situations: (1) driving straight along a 4-lane highway in a rural environment where local lanes are divided by a barrier, traffic conditions are low and there is good weather, and (2) driving straight along on a 3-lane urban road with heavy traffic, high pedestrian flow, and where traffic lights are expected. The two scenarios were based on scenarios used in a NHTSA report investigating voluntary distractions (Lerner \& Boyd, 2005) and were modified for the purpose of our research focus. A written description of each scenario is presented alongside an image of a driving environment with these characteristics. 


\section{HABITUAL ENGAGEMENT IN DRIVER DISTRACTION}

Habits are defined as "a form of automaticity in responding that develops as people repeat actions in stable circumstances", (Verplanken \& Wood, 2006). These actions are goal-oriented behaviours that were originally motivated by an expected reward, but are now performed without deliberate behavioural intention. Such actions can be triggered directly by the perception of the situational cues that were, in the past, contiguous with the behaviour (Verplanken $\&$ Wood, 2006). These triggers can include environmental cues, such as time of day and location (Wood \& Neal, 2007) or internal mental states, such as a particular mood (Ji \& Wood, 2007).

The new questionnaire explores habitual engagement in driver distraction as certain behaviours around cell phone use may have become automatic due to the prevalence of mobile technology in our everyday life. For example, glancing at the mobile phone to check for updates, which is goal-oriented to start, may have become a habit for many in various contexts and environments. Research has suggested that texting while driving may be partially accounted by habitual tendencies in which drivers perform the behaviour without awareness, control, attention, or intention to do so (Bayer \& Campbell, 2012). Importantly, these findings remained significant after holding conscious predictors (i.e., attitudes, perceived behavioural control, and social norms) constant. However, we note that habitual engagement may be associated with controllability captured through the TPB framework, an aspect of perceived behavioural control that examines drivers' beliefs about their ability to control if and when they engage in distractions. Given that habits are executed with limited control and conscious awareness, it would be interesting to explore the relationships between these two constructs. Data collected using this questionnaire may help clarify whether habits influence behaviours directly or indirectly through perceived behavioural control in the context of cell phone use.

In our revision of SDDQ, we adopt the Self-Report Habit Index (SRHI; Verplanken \& Orbell, 2003 ) to evaluate habitual cell phone-related behaviours, such as answering a phone call or responding to notifications. It is important to note that SRHI does not associate frequency of engagement in a behaviour with habit strength. Instead, it captures strength of a habit based on the features that characterize the general experience of habits: uncontrollability, execution without awareness, efficiency, and identity. Example items that are used in the new questionnaire include "answering a phone call is something I do automatically" and "texting is something that is typically 'me'".

\section{INVOLUNTARY ENGAGEMENT IN DRIVER DISTRACTION}

Involuntary driver distraction occurs as a result of drivers' inability to ignore distracting stimuli due to the physical or psychological properties that make the stimuli highly salient, such as being unusual, irritating, unexpected, or sudden (Regan, Hallett, \& Gordon, 2011). Despite the fact that salient stimuli will capture attention automatically (Franconeri \& Simons, 2003), drivers should recognize stimuli that are irrelevant to the driving task and refocus their attention to driving. The ability and speed at which individuals can disengage from a previously attended stimulus varies with cognitive abilities (Cosman, Lees, Lee, Rizzo, \& Vecera, 2011). In order to capture involuntary distraction, the current questionnaire examines items in which drivers will report on the difficulty associated with ignoring common distractions, such as roadside accident scenes or 
alerts on cell phones that they do not intend to respond to. In addition, the current questionnaire probes drivers on how often they find themselves engaging in non-driving related tasks for longer than intended or realized (including being lost in thought), as an indicator of their ability to disengage their attention from these tasks.

\section{CONCLUDING REMARKS}

This paper describes revisions and new additions to the Susceptibility to Driver Distraction Questionnaire (SDDQ) to address the limitations previously identified. In particular, habitual engagement, a third category of driver distraction in addition to voluntary and involuntary engagement has been identified and included in the revised questionnaire. In an upcoming online survey study, the 400-item questionnaire discussed in this paper will be administered to explore the effectiveness of these items in measuring susceptibility to voluntary, involuntary, and habitual distraction. Using factor analyses and reliability statistics, we aim to determine the best set of items to be included in the next generation of SDDQ. Ultimately, the goal of SDDQ is to provide a reliable and valid means of understanding individual differences in driver distraction, which may lead to better design of countermeasures for driver distraction.

\section{ACKNOWLEDGEMENTS}

Funding for this work was provided by the Toyota Collaborative Safety Research Center (CSRC) and Auto21 Network of Centres of Excellence. Many thanks to James Foley, Kazu Ebe, and Chuck Gulash from Toyota CSRC for their feedback on this paper as well as providing directions for our research in general.

\section{REFERENCES}

Ajzen, I. (1991). The Theory of Planned Behavior. Organizational Behavior and Human Decision Processes, 50(2), 179-211.

Bayer, J. B., \& Campbell, S. W. (2012). Texting while driving on automatic: Considering the frequency-independent side of habit. Computers in Human Behavior, 28(6), 2083-2090.

Chen, H. Y. W., Donmez, B., Hoekstra-Atwood, L., \& Marulanda, S. (n.d.). Self-reported engagement in driver distraction: An application of the Theory of Planned Behaviour. Transportation Research Part F: Traffic Psychology and Behaviour.

Cosman, J. D., Lees, M. N., Lee, J. D., Rizzo, M., \& Vecera, S. P. (2011). Impaired attentional disengagement in older adults with useful field of view decline. The Journals of Gerontology, Series B: Psychological Sciences and Social Sciences, 67(4), 405-412.

Dingus, T. A., Klauer, S. G., Neale, V. L., Petersen, A., Lee, S. E., Sudweeks, J., ... Knipling, R. R. (2006). The 100-Car Naturalistic Driving Study, Phase II-Results of the 100-Car Field Experiment. DOT HS 810 593. Washington, DC: National Highway Traffic Safety Administration.

Feng, J., Marulanda, S., \& Donmez, B. (2014). Susceptibility to Driver Distraction Questionnaire (SDDQ): Development and relation to relevant self-reported measures. Transportation Research Record, 2423, 26-34.

Franconeri, S., \& Simons, D. (2003). Moving and looming stimuli capture attention. Perception and Psychophysics, 65(7), 999-1010. 
Ji, M., \& Wood, W. (2007). Purchase and consumption habits: Not necessarily what you intend. Journal of Consumer Psychology, 17(4), 261-276.

Klauer, S. G., Guo, F., Simons-Morton, B. G., Ouimet, M. C., Lee, S. E., \& Dingus, T. A. (2014). Distracted driving and risk of road crashes among novice and experienced drivers. The New England Journal of Medicine, 370(1), 54-59.

Lerner, N., \& Boyd, S. (2005). Task Report: On-Road Study of Willingness to Engage in Distracting Tasks. DOT HS 809 863. Washington, DC: National Highway Traffic Safety Administration.

Marulanda, S., Chen, H. Y. W., \& Donmez, B. (2015). Test-retest reliability of the Susceptibility to Driver Distraction Questionnaire (SDDQ). In Transportation Research Board 94th Annual Meeting. Washington, DC.

McEvoy, S. P., Stevenson, M. R., \& Woodward, M. (2006). The impact of driver distraction on road safety: Results from a representative survey in two Australian states. Injury Prevention, 12(4), 242-247.

National Highway Traffic Safety Administration. (2014). Distracted Driving 2012. DOT HS 812 012. Washington, DC: National Highway Traffic Safety Administration.

Regan, M. A., Hallett, C., \& Gordon, C. P. (2011). Driver distraction and driver inattention: Definition, relationship and taxonomy. Accident Analysis and Prevention, 43(5), 1771-1781.

Schroeder, P., Meyers, M., \& Kostyniuk, L. (2013). National Survey on Distracted Driving, Attitudes and Behaviors. DOT HS 811 729. Washington, DC: National Highway Traffic Safety Administration.

Strayer, D. L., Drews, F. A., \& Johnston, W. A. (2003). Cell phone-induced failures of visual attention during simulated driving. Journal of Experimental Psychology: Applied, 9(1), 2332.

Strayer, D. L., Watson, J., \& Drews, F. (2011). Cognitive distraction while multitasking in the automobile. In B. H. Ross (Ed.), The Psychology of Learning and Motivation (Vol. 54, pp. 29-58). Burlington: Academic Press.

Tison, J., Chaudhary, N., \& Cosgrove, L. (2011). National Phone Survey on Distracted Driving Attitudes and Behaviors. DOT HS 811 555. Washington, DC: National Highway Traffic Safety Administration.

Verplanken, B., \& Orbell, S. (2003). Reflections on past behavior: A self-report index of habit strength. Journal of Applied Social Psychology, 33(6), 1313-1330.

Verplanken, B., \& Wood, W. (2006). Interventions to break and create consumer habits. Journal of Public Policy and Marketing, 25(1), 90-103.

Wood, W., \& Neal, D. T. (2007). A new look at habits and the habit-goal interface.

Psychological Review, 114(4), 843-863. 